\title{
16. PRELIMINARY DATING BY FOSSIL CALCAREOUS NANNOPLANKTON DEEP SEA DRILLING PROJECT, LEG 1
}

\author{
W.W. Hay, Institute of Marine Sciences, University of Miami, Miami, Florida
}

Throughout this report samples are referred to in the same manner as in Chapter 15. The sample numbers indicate the relative positions of the samples in the holes and consist of the following sequence: (cruiseleg number) - (drill-hole designation, consisting of site number plus a letter if more than one hole) - (core number) - (core section number). This series is followed by the interval below the top of each core section in centimeters from which the sample was cut.

\section{PISTON CORE, SITE 1}

Sample 1-1P-1-1, $20 \mathrm{~cm}$ :

Sample 1-1P-1-1, $78 \mathrm{~cm}$ :

These samples contain the typical modern assemblage of calcareous nannoplankton for this area: Emiliania huxleyi; Gephyrocapsa oceanica; Helicopontosphaera scutellum; and, Cyclolithella annula. In addition, a new species known from the Caribbean to become abundant at the level oxygen isotope ratios would indicate to be the end of Wisconsinan Glaciation, is very abundant in these samples also.

Age: Late Emiliania huxleyi Zone; post Wisconsinan (= Recent).

(Note: Very few reworked Cretacoeus coccoliths are present in these samples.)

Sample 1-1P-1-1, $140 \mathrm{~cm}$ :

Sample 1-1P-1-2, $77 \mathrm{~cm}$ :

Sample 1-1P-1-2, $138 \mathrm{~cm}$ :

Sample 1-1P-1-3, $120.5-121.5 \mathrm{~cm}$ :

Sample 1-1P-1-4, $2 \mathrm{~cm}$ :

Sample 1-1P-1-5, 141 - $142 \mathrm{~cm}$ :

The samples contain the same assemblage as those listed above. Changes in abundance suggest that these sediments might be Wisconsinan. Silt and Cretaceous coccoliths are increasingly abundant in the lower samples, although that from Section 4 yields a good indigenous assemblage.

Age: Emiliania huxleyi Zone; perhaps Wisconsinan (= late Calabrian).

Comments: The rich modern calcareous nannoplankton assemblages of the upper part of Section 1 are a striking contrast to the sterile drilled cores. The lower part of the piston core represents the transition between the two types of sediment, and might correspond to a shift in the mouth of the Mississippi from a southerly discharge to an easterly discharge.

\section{HOLE 1}

Careful concentration of the nannoplankton size fraction (2-30 microns) was attempted for the following samples:

1-1-1-3, 2-3 cm:

1-1-1-6, 3-4 cm:

$1-1-2-2,6.5-7.5 \mathrm{~cm}$ :

1-1-5-1, 7-8 cm:

1-1-6-1, 13-14 cm:

$1-1-7-2,7.5-8.5 \mathrm{~cm}$ :

1-1-7-6, 6-7 cm:

1-1-8-2, 9-10 cm:

$1-1-8-3,9-10 \mathrm{~cm}$ :

1-1-9-2, 12-13 cm:

1-1-9-5, 9-10 cm:

1-1-9-7, 9-10 cm:

Calcareous nannoplankton fossils are extremely rare in all of these samples, and only Cretaceous species were found. The most common nannofossils are Micula staurophora (Gardet) and Prediscosphaera cretacea (Arkhangelskii). All of the nannofossils are interpreted as reworked.

The complete absence of indigenous Pleistocene or Recent nannoplankton fossils is striking. Preservation of the Cretaceous specimens is fair to good, being about the same as that in their probable source area, the Cretaceous of the Great Plains. A possible explanation of the absence of contemporary nannofossils in this section might be that the Mississippi waters were directed out over this area during the time of deposition, preventing the growth of calcareous nannoplankton in the surface waters.

\section{HOLE 2}

Sample 1-2-1-2, 4-5 cm:

Sample 1-2-1-3, 4-5 cm:

These samples contain rich assemblages with common Cyclococcolithus leptoporus, Umbilicosphaera mirabilis, Helicopontosphaera kamptneri, Ceratolithus cristatus and other species common in Pleistocene samples.

Stratigraphically important are: Gephyrocapsa caribbeanica; Gephyrocapsa aperta; and Gephyrocapsa oceanica.

Age: Early Gephyrocapsa oceanica Zone; probably early glacial Pleistocene (= mid-Calabrian). 
Sample 1-2-2-1, 41-42 cm:

Contains Helicopontosphaera kamptneri; Discolithina anisotrema; Rhabdosphaera clavigera; Cyclococcolithus leptoporus; Umbilicosphaera mirabilis; Scyphosphaera apsteini; Discoaster brouweri; Discoaster brouweri rutellus; Discoaster triradiatus.

Age: Discoaster brouweri Zone; probably "Nebraskan" of Gulf Coast; latest Piacenzian; late Pliocene.

Sample 1-2-3-2, $3 \mathrm{~cm}$ :

Sample 1-2-3-3, 5-6 cm:

Sample 1-2-3-3, 75-76 cm:

Contain same assemblage as above, plus Discoaster pentaradiatus; Discoaster extensus.

Age: Discoaster extensus Zone; late Piacenzian; late Pliocene.

Sample 1-2-4-1, 36-37 cm:

Sample 1-2-42, 6-7 cm:

Contain Cyclococcolithus leptoporus; Helicopontosphaera kamptneri; "Coccolithus" pseudoumbilicus; Discoaster brouweri; Discoaster extensus; and, Discoaster sp. aff. D. surculus.

Age: Top of Discoaster surculus Zone; Piacenzian, but younger than stratotype.

\section{HOLE 3}

Sample 1-3-1-2, 24-25 cm:

Sample 1-3-1-2, 74.5-75.5 cm:

Very poor assemblages; mostly reworked Cretaceous species with some Pleistocene species admixed.

No accurate age determination possible at present.

Sample 1-3-2-3, 1.5-2.5 cm:

Reworked Cretaceous species are common, but Gephyrocapsa oceanica is present.

Age: Gephyrocapsa oceanica Zone or younger; glacial Pleistocene; mid-Calabrian or younger.

Sample 1-3-3-2, $108 \mathrm{~cm}$ :

Some reworked Cretaceous species, but essentially a coccolith ooze, with Cyclococcolithus leptoporus; Helicopontosphaera kamptneri; Umbilicosphaera mirabilis; Rhabdosphaera stylifera; Gephyrocapsa oceanica; and, Discosphaera tubifera.

Age: High in Gephyrocapsa oceanica Zone (IllinoisianSangamon???); Mid-Calabrian.

Sample 1-3-1, $50 \mathrm{~cm}$ :

Contains same assemblage as in Core 3 , except Discosphaera tubifera was not found, and Gephyrocapsa caribbeanica and Gephyrocapsa aperta are common. Age: Low in Gephyrocapsa oceanica Zone; midCalabrian.

Sample 1-3-5-1, $33 \mathrm{~cm}$ :

Sample 1-3-5-2, $1 \mathrm{~cm}$ :

Sample 1-3-5-3, 9-10 cm:
Sample 1-3-5- $10 \mathrm{~cm}$ :

Contains Cyclococcolithus leptoporus; Helicopontosphaera kamptneri; Scyphosphaera apsteini; Scyphosphaera cf. conica; Rhabdosphaera stylifera; Discoaster brouweri; Discoaster pentaradiatus; Discoaster surculus; and many species of Pontosphaera.

Age: High in Discoaster surculus Zone, younger than type Piacenzian; Upper Pliocene.

Sample 1-3-6-1, 45.5-47 cm:

Sample 1-3-6-2, 2-3 cm:

Calcareous nannofossils are heavily calcified; specific determinations are difficult in some cases, but the assemblage is essentially like that in Core 5 .

Age: High in Discoaster surculus Zone; Piacenzian, but younger than type; Upper Pliocene.

Sample 1-3-7-1, $7 \mathrm{~cm}$ :

Sample 1-3-7-2, 0-1 cm:

Sample 1-3-7-3, $78 \mathrm{~cm}$ :

Sample 1-3-7-4, 4-5 cm:

Assemblages are poor, but contain Discoaster brouweri; Discoaster pentaradiatus; Discoaster perplexus; Discoaster triradiatus; Scyphosphaera apsteini; Helicopontosphaera kamptneri; Cyclococcolithus leptoporus; and very rare Discoaster surculus. The occurrence of fourrayed specimens of Discoaster brouweri in these samples may be significant in a more precise determination of the age of the sediments.

Age: Discoaster surculus Zone, near top; Piacenzian, but younger than stratotype; Upper Pliocene.

Sample 1-3-8-2, $7 \mathrm{~cm}$ :

Sample 1-3-8-3, 7-8 cm:

Sample 1-3-8-4, 7-8 cm:

Sample 1-3-8-6, 8-9 cm:

Very rich assemblages, dominated by Discoaster pentaradiatus and Discoaster surculus. Other common species include "Coccolithus" pseudoumbilicus; Discoaster brouweri; and Cyclococcolithus leptoporus. A few three-rayed specimens of Discoaster surculus are present, along with Ceratolithus cf. tricorniculatus and Ceratolithus cristatus.

Age: Discoaster surculus Zone, lower part; very similar to type Piacenzian, but might be "Zanclian" of Blow.

Sample 1-3-9-2, 0-1 cm:

Sample 1-3-9-3, 0-1 cm:

Contain Discoaster brouweri; Discoaster pentaradiatus;

Discoaster extensus; and an unnamed discoaster which might be an immediate forerunner of Discoaster surculus. Age: Late Messinian.

Sample 1-3-9-4, 3-4 cm:

Barren.

Sample 1-3-9-5, 0-1 cm:

Similar to Sections 2 and 3 of Core 9. 
Sample 1-3-9-5, $75 \mathrm{~cm}$ :

Sample 1-3-9-6, 0-1 cm:

Sample 1-3-9-7, 4-5 cm:

Contains Discoaster brouweri; Discoaster brouweri rutellum; Discoaster extensus; Discoaster variabilis; Discoaster bollii; and, six-rayed specimens of Discoaster hamatus.

Age: Late Tortonian or early Messinian.

Sample 1-3-10-2, 8-9 cm:

Barren.

Sample 1-3-10-2, $100 \mathrm{~cm}$ :

Poor assemblage containing Discoaster brouweri; Discoaster brouweri rutellus; Discoaster calcaris; Discoaster hamatus??; Discoaster stellulus??.

Age: Late Tortonian or early Messinian.

Sample 1-3-11-1, 53-54 cm:

Very sandy sample, apparently a turbidite layer. Contains Micula staurophora; Discoaster barbadiensis; Discoaster brouweri; Discoaster deflandrei; mixed assemblage.

Age: Langhian or Tortonian, precise determination not possible.

\section{HOLE 4}

Sample 1-4-1-1, $20 \mathrm{~cm}$ :

Barren.

Sample 1-4-1-2, 0-2 cm:

Contains Discoaster dilatus; Discoaster nephados; Cyclococcolithus leptoporus; Discoaster brouweri; Spenolithus spp.

Age: Burdigalian; late-early Miocene.

(Note: Cretaceous species are admixed.)

Sample 1-4-1-3, 3-4 cm:

Barren.

Sample 1-4-1-4, 0-2 cm:

Similar to Section 2.

Sample 1-4-1-5, 0-2 cm:

Barren.

Sample 1-4-1-6, 0-2 cm:

Barren.

Sample 1-4-2-1, $9 \mathrm{~cm}$ :

Contains Eiffellithus turriseiffeli; Micula staurophora; Tetralithus pyramidus; Microrhabdulus decoratus; and, Marthasterites furcatus.

Age: Late Tortonian or Coniacian.

Sample 1-4-2-2, 0-2 cm:

Contains Eiffellithus turriseiffeli; but lacks Micula staurophora and Microrhabdulus decoratus.

Age: Late Cenomanian or early Turonian.

Sample 1-4-3-1, 10-11 cm:

Well preserved, interesting assemblage. Most species not yet described, but "Zygolithus" striatus and Cricolithus multi radiatus occur.

Age: Probably Aptian or Albian.

Sample 1-4-4-1, 10-11 cm:

Contains Parhabdolithus embergeri; Watznaueria barnesae; Octopodorhabdus praevisus; Diazomatolithus lehmani, Cyclagelosphaera margereli; and, Staurolithites sp.

Age: Probably Kimmeridgian or Portlandian.

\section{HOLE 4A}

Sample 1-4A-1-1, 5-6 cm:

Preservation very poor. Cribrosphaera murrayi; Cribrosphaera ehrenbergi; Prediscosphaera cretacea; Arkhangelskiella parca; Microrhabdulus decoratus; Micula staurophora; Eiffellithus turiseiffeli; Lithraphidites carniolensis; and, Tetralithus aculeus are present.

Age: Campanian.

Sample 1-4A-1-2, 0-1 cm:

Barren.

Sample 1-4A-1-3, 9-10 cm:

Very poor preservation, sparse assemblage like that in Section 1.

Sample 1-4A-2-1, 10-11 cm:

Barren.

\section{HOLE 5}

Sample 1-5-1-1, 0-2 cm:

Contains Gephyrocapsa oceanica along with much reworked material from the Miocene and Eocene.

Age: Gephyrocapsa oceanica Zone or younger; glacial Pleistocene or Recent; late Calabrian or Recent.

Sample 1-5-1-2, 0-2 cm:

Contains Discoaster nephados; Discoaster perplexus; and, Coccolithus eopelagicus along with many reworked specimens from the Upper Cretaceous.

Age: Probably Burdigalian, late Lower Miocene.

Sample 1-5-3-1, 9-10 cm:

Preservation very poor; contains Micula staurophora and Eiffellithus turriseiffeli.

Age: Turonian or younger.

Sample 1-5-6, Core catcher:

Contains very abundant Nannoconus steinmanni; also Diazomatolithus lehmani; Cyclagelosphaera margereli; Parhabdolithus embergeri; Watznaueria barnesae.

Age: Tithonian. 


\section{HOLE 6}

Sample 1-6-2-1, 4-6 cm:

Sample 1-6-3-3, $2 \mathrm{~cm}$ :

Sample 1-6-3-4, 6-7 cm:

Sample 1-6-4-2, $12 \mathrm{~cm}$ :

Sample 1-6-4-3, 5-6 cm:

Sample 1-6-6-3, 9-10 cm:

These samples contain rich, well preserved calcareous nannofossil assemblages along with much diatom and radiolarian material. The most abundant species are: Chiasmolithus solitus; Neococcolithes dubius; Chiasmolithus expansus; Discoaster barbadiensis; Discoaster gemmifer; Discoaster tani tani; and, Discoaster saipanensis.

Age: Late Chiphragmalithus quadratus Zone; late Lutetian; late Middle Eocene.

(Note: The Chiphragmalithus quadratus Zone is the interval between the first occurrence of the name species and the first occurrence of Discoaster tani nodifer. It is surprising that this interval should be so thick, but this part of the Eocene is very poorly known in pelagic facies.)

\section{HOLE 7}

Sample 1-6-1-1, 0+ cm:

Contains Cyclococcolithus leptoporus; Ceratolithus cristatus; and, abundant very small coccoliths, many of which are probably Gephyrocapsa caribbeanica. Gephyrocapsa oceanica is not present.

Age: Gephyrocapsa caribbeanica Zone; early Pleistocene, early Calabrian.
Sample 1-6-1-2, 8-9 cm:

Contains Discoaster brouweri and Discoaster pentaradiatus.

Age: Discoaster brouweri Zone; latest Piacenzian; latest Pliocene.

Sample 1-6-1-3, 3-4 cm:

Contains poor assemblage with Apertapetra umbilica; Chiasmolithus bidens; Discoaster barbadiensis.

Age: Upper or Middle Eocene.

(Note: This sample is probably either contaminated or mislabeled.)

Sample 1-6-1-4, 8-10 cm:

Contains Ceratolithus cristatus; Cyclococcolithus leptoporus; Discoaster triradiatus; Discoaster brouweri rutellus; Discoaster pentaradiatus; Umbilicosphaera mirabilis; "Discolithus" antillarum.

Age: Discoaster brouweri Zone; latest Piacenzian; latest Pliocene.

Sample 1-6-2, Core catcher:

Contains Chiasmolithus grandis; Apertapetra umbilica; Discoaster barbadiensis; Coccolithus eopelagicus.

Age: Middle or Upper Eocene.

\section{HOLE 7A}

Sample 1-7-2-1, 12-14 cm:

Barren. 